\title{
Optically Enhanced Electron Attachment
}

\author{
L. G. Christophorou, ${ }^{(a)}$ S. R. Hunter, L. A. Pinnaduwage, ${ }^{(a)}$ J. G. Carter, A. A. Christodoulides, ${ }^{(b)}$ \\ and S. M. Spyrou (c) \\ Atomic, Molecular, and High Voltage Physics Group, Health and Safety Research Division, \\ Oak Ridge National Laboratory, Oak Ridge, Tennessee 37831 \\ (Received 23 September 1986)
}

\begin{abstract}
The first optically enhanced electron attachment involving electronically excited molecules is reported. The metastable (triplet) states responsible for the photoenhanced electron attachment were produced indirectly via internal conversion from higher excited singlet states which are strongly optically allowed and are reached by excimer-laser excitation.
\end{abstract}

PACS numbers: $34.80 . G s, 33.80 .-b$

Slow-electron-excited-molecule interactions have been scarcely studied in spite of their basic and applied significance. ' Large changes in the cross section for negative-ion formation have been observed when slow electrons collide with molecules excited thermally into vibrational-rotational states of the electronic ground state (see Ref. 1 and references given therein). Enhanced dissociative attachment via electron capture by vibrationally excited molecules produced by laser irradiation has also been reported (e.g., for $\mathrm{SF}_{6}{ }^{2}$ and $\mathrm{I}_{2}{ }^{3}$ ). Additionally, enhanced electron attachment to vibrationally excited $\mathrm{HCl}$ and $\mathrm{HF}$ molecules produced, respectively, by laser photodissociation of the $\mathrm{C}_{2} \mathrm{H}_{3} \mathrm{Cl}$ and $\mathrm{C}_{2} \mathrm{~F}_{3} \mathrm{H}$ molecules has been observed. ${ }^{4}$ To our knowledge, the only studies on electron attachment to electronically excited molecules are the experiments ${ }^{5}$ on dissociative attachment to $\mathrm{O}_{2}\left(a^{1} \Delta_{\mathrm{g}}\right)$ produced in a microwave discharge and the calculation ${ }^{6}$ of the dissociativeattachment cross section for $\mathrm{H}_{2}\left(c^{3} \Pi_{\mathrm{u}}\right)$; both of these studies indicated larger attachment cross sections for the electronically excited molecule compared with the ground-state molecule. In this paper we report the first observation of optically enhanced electron attachment involving electronically excited molecules.

The principle of the present experiment and the required properties of the electron-attaching molecules, $A X$, are shown in Fig. 1. The electron-attachment properties of $A X$-present in minute quantities in a buffer gas $\left(\mathrm{N}_{2}\right.$ in the present study) - were first studied without laser excitation with the use of two different electronswarm methods: the pulse-shape method $^{7}$ and a modified pulsed Townsend technique. ${ }^{8}$ Subsequently, an excimer laser was used to excite via single-photon absorption a strongly allowed electronic singlet state, $S_{n}$, of $A X\left[A X\right.$ (ground-state singlet, $S_{0}$ ) $+h v \rightarrow A X^{* *}$ (highly allowed second or third excited singlet state, $\left.S_{2 / 3}\right)$ ] which lies below the lowest excited electronic state of $\mathrm{N}_{2}$. The $A X^{* *}\left(S_{2,3}\right)$ molecule normally undergoes fast $\left(\sim 10^{-13} \mathrm{~s}\right)$ intramolecular relaxation to its first excited singlet state, $S_{1}$,

$$
A X^{* *}\left(S_{2,3}\right) \stackrel{-10^{13} \mathrm{~s}}{\rightarrow} A X^{*}\left(S_{1}\right)
$$

except when dissociation into neutral fragments, which depends on the photon wavelength, also occurs. The $A X^{*}\left(S_{1}\right)$ species, then, undergoes rapid (within $\sim 10^{-8}$ to $10^{-11} \mathrm{~s}$, depending on $A X$ ) "intersystem crossing" to the lowest triplet state, $T_{1}$,

$$
A X^{*}\left(S_{1}\right) \stackrel{10^{-8}-10^{-11} \mathrm{~s}}{\rightarrow} A X^{*}\left(T_{1}\right) \text {. }
$$

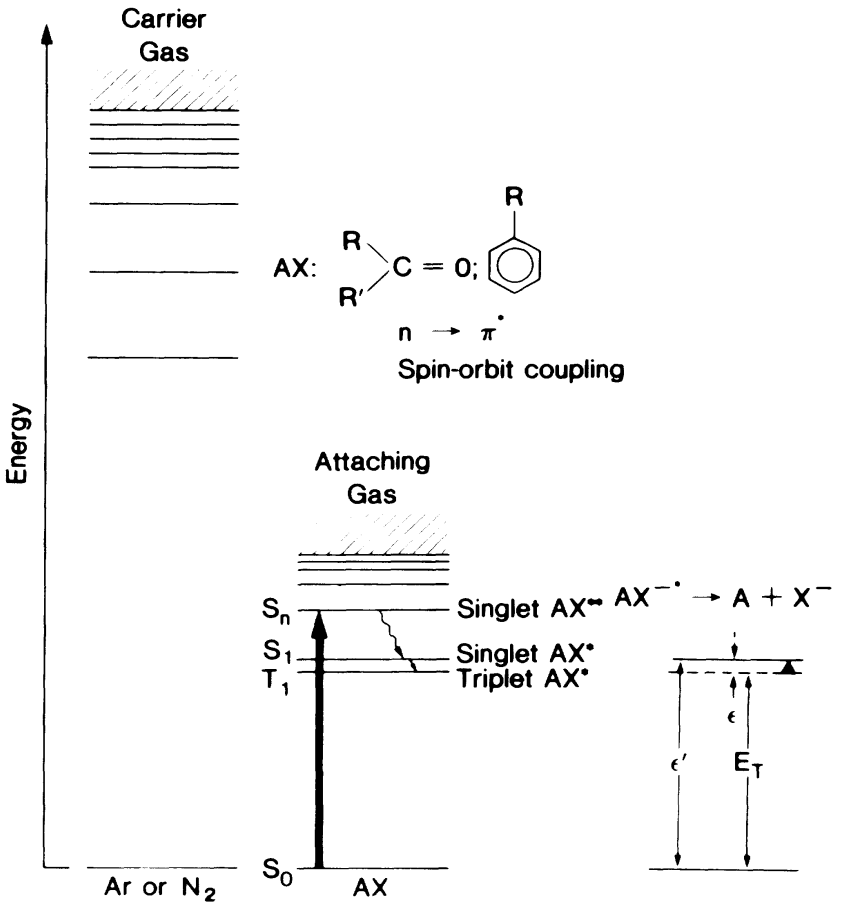

FIG. 1. Schematic energy-level diagram of carrier- and attaching-gas system. A possible electron-attachment mechanism from the triplet state $A X^{*}\left(T_{1}\right)$ (dissociative electron attachment) is shown. While this mechanism may contribute slightly to the observed photoenhanced attachment (and might be the dominant mechanism for other molecules), for the molecules investigated, the observed photoenhanced attachment is attributed predominantly to a nondissociative electron-attachment process involving $A X^{*}\left(T_{1}\right)$ and another ground-state molecule, $A X\left(S_{0}\right)$. 
The excited $A X^{*}\left(T_{1}\right)$ molecule remains in the $T_{1}$ state for times $\gtrsim 1 \mathrm{~ms}$, which allows ample time for collisions with slow electrons. While we expected (see Fig. 1) that electrons would be captured dissociatively into a negative-ion state from $T_{1}$, viz.,

$$
A X^{*}\left(T_{1}\right)+e(\varepsilon) \rightarrow A X^{*-} \rightarrow A+X^{-},
$$

the present findings (see below) indicate that, for the molecules studied, electron capture is nondissociative and, instead of reaction (1), it involves $A X^{*}\left(T_{1}\right)$ and another ground-state molecule, $A X\left(S_{0}\right)$, viz.,

$$
A X^{*}\left(T_{1}\right)+A X\left(S_{0}\right)+e \rightarrow \text { anions. }
$$

We have identified two classes of molecules which generally possess the aforementioned characteristics and might thus exhibit optically enhanced electron attachment, namely, certain benzene derivatives ${ }^{9,10}$ and certain carbonyl compounds. ${ }^{9-12}$ We have selected thiophenol $\left(\mathrm{C}_{6} \mathrm{H}_{5} \mathrm{SH}\right)$ and thioanisole $\left(\mathrm{C}_{6} \mathrm{H}_{5} \mathrm{SCH}_{3}\right)$ as representative candidate molecules from the first group. For both molecules we have observed large optically enhanced electron attachment. Our initial studies have focused on $\mathrm{C}_{6} \mathrm{H}_{5} \mathrm{SH}$.

The present experiments were performed as follows: The electron-attaching gas was mixed in small amounts $(0.0013-0.013 \mathrm{kPa})$ with $\mathrm{N}_{2}(66.6-266.6 \mathrm{kPa})$ and was studied with and without laser irradiation. In the former case the interelectrode space $(2 \mathrm{~cm}$ in length and $\sim 1$ $\mathrm{cm}^{2}$ cross-sectional area) was irradiated with a laser beam through a gridded anode; this same laser beam, at the end of its path, was absorbed by the cathode and photoelectrically injected a pulse of electrons into the gas which drifted-as an electron swarm-toward the anode, colliding en route with $A X^{*}\left(T_{1}\right)$ produced in the irradiated gas. Since the electron drift times are $\sim 10^{-6}$ s, virtually all $A X^{*}\left(T_{1}\right)$ molecules remain excited during the entire transit time of the electron swarm. The electron-attachment measurements were made at various laser-pulse energies $(0.05-5 \mathrm{~mJ})$ and for the following laser wavelengths: $308 \mathrm{~nm}(4.03 \mathrm{eV}, \mathrm{XeCl}) ; 249 \mathrm{~nm}$ $(4.98 \mathrm{eV}, \mathrm{KrF}) ; 222 \mathrm{~nm}(5.59 \mathrm{eV}, \mathrm{KrCl})$; and $193 \mathrm{~nm}$ (6.42 eV, ArF). Absorption-spectrum measurements of $\mathrm{C}_{6} \mathrm{H}_{5} \mathrm{SH}$ in liquid iso-octane ${ }^{13}$ indicate that the approximate position of the first excited triplet state $T_{1}$ (onset of phosphorescence) ${ }^{14}$ is $3.35 \mathrm{eV}$ and that of the first excited singlet state ( $0-0$ transition) is $4.36 \mathrm{eV} .^{13,14}$

In Fig. 2 are shown the measured electron-attachment coefficients, $\eta$, normalized to the ground-state attaching-gas number density, $N_{a}, \eta / N_{a}$, versus $E / N$ ( $E$ is the electric field strength and $N$ the total gas number density) for $\mathrm{C}_{6} \mathrm{H}_{5} \mathrm{SH}$. Curve 1 was obtained without irradiation; curve 2 was obtained with $308-n m$ laser-light irradiation for a laser-pulse energy of $\sim 1 \mathrm{~mJ}$. Both sets of data are similar; the $\eta / N_{a}$ versus $E / N$ functions for both curves 1 and 2 peak at a mean electron energy of $\sim 0.8$ $\mathrm{eV}$ and are $<5 \times 10^{-19} \mathrm{~cm}^{2}$ (the detection threshold of

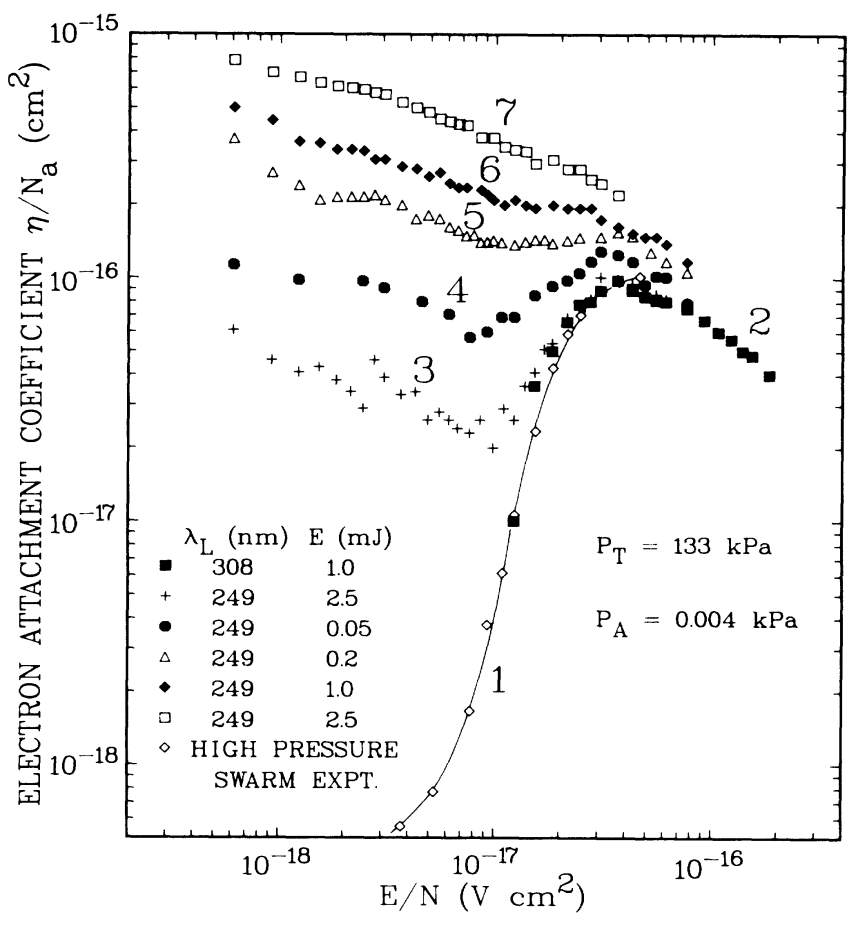

FIG. 2. Electron-attachment coefficient, $\eta / N_{a}$, vs $E / N$ for $\mathrm{C}_{6} \mathrm{H}_{5} \mathrm{SH}$ in $\mathrm{N}_{2}$ for the ground-state (curves 1 and 2) and the electronically excited (curves $3-7$; curve 3 is for a single laser pulse, and curves 4-7 are for the double laser pulses $\mathrm{C}_{6} \mathrm{H}_{5} \mathrm{SH}$ molecules.

the present technique) at low energies (low $E / N$ ). These data are consistent with the results that we obtained from a mass-spectrometric study on unexcited $\mathrm{C}_{6} \mathrm{H}_{5} \mathrm{SH}$ which indicated that the most abundant negative ion is $\mathrm{C}_{6} \mathrm{H}_{5} \mathrm{~S}^{-}$with an onset energy of $0.7 \mathrm{eV}$ and a peak in the attachment cross section at $1.3 \mathrm{eV}$. These results are also consistent with electron-transmission studies which indicated ${ }^{15}$ that the vertical attachment ener$\mathrm{gy}^{7}$ for the lowest $\pi^{*}$ negative-ion state of $\mathrm{C}_{6} \mathrm{H}_{5} \mathrm{SH}$ lies in the range 0.7 to $1.1 \mathrm{eV}$. The $308-\mathrm{nm}$ light does not appreciably monophotonically excite $\mathrm{C}_{6} \mathrm{H}_{5} \mathrm{SH}$, and unless the laser-pulse energy is high enough for multiphoton absorption to occur, electron attachment involves only ground-state $\mathrm{C}_{6} \mathrm{H}_{5} \mathrm{SH}$ molecules. The rest of the data in Fig. 2 were taken with the $\mathrm{KrF}$ laser line (249 $\mathrm{nm})$. The curve- 3 measurements were taken with a single laser pulse of $2.5-\mathrm{mJ}$ energy and show that $\eta / N_{a}$ above $E / N \sim 1 \times 10^{-17} \mathrm{~V} \mathrm{~cm}{ }^{2}$ is due to electron capture by ground-state molecules and below $E / N \sim 0.5 \times 10^{-17}$ $\mathrm{V} \mathrm{cm}^{2}$ (corresponding to a mean electron energy of $\sim 0.2 \mathrm{eV}$ ) is substantially due to electron capture by electronically excited molecules. Much larger photoenhanced $\eta / N_{a}$ values were measured when a second similar (of the same energy) laser pulse followed the first at time intervals between 12.5 and $900 \mathrm{~ms}$. The curves 4 to 7 in Fig. 2 are such measurements for a time delay, 
$\tau_{d}$, between the two similar laser pulses of $12.5 \mathrm{~ms}$ and for the pulse energies shown in the figure.

The observed photoenhanced attachment involves $A X^{*}\left(T_{1}\right)$ and is consistent with a nondissociativeelectron-attachment mechanism involving $A X^{*}\left(T_{1}\right)$ and $A X\left(S_{0}\right)$, as for example,

$$
\begin{aligned}
& A X^{*}\left(T_{1}\right)+A X\left(S_{0}\right) \rightarrow \text { triplet excimer }(?), \\
& \text { triplet excimer }+e \stackrel{\mathrm{N}_{2}}{\rightarrow} \text { anions, }
\end{aligned}
$$

for the following reasons:

(1) The photoenhanced $\eta / N_{a}$ is very much larger for the second than for the first laser pulse. This would indicate that the excited species $A X^{*}\left(T_{1}\right)$ produced by the first laser pulse does not significantly attach the electrons produced by that laser pulse [e.g., via reaction (1)] but rather reacts with an unexcited $A X\left(S_{0}\right)$ molecule to form the electron-attaching species (possibly a tripletstate excimer). ${ }^{16}$ For the $N_{a}$ densities used, this would take times of the order of $10^{-6} \mathrm{~s}$, and thus few species would be formed during the first laser pulse, and many more would be present to attach the electrons produced by the second laser pulse.

(2) The photoenhanced $\eta / N_{a}$ decreases with increasing $\tau_{d}$ between the two laser pulses (for $\tau_{d} \geq 12.5 \mathrm{~ms}$ ) indicating diffusion (or decay) of the electron-attaching species outside of the path of the second laser pulse (in this regard studies with shorter $\tau_{d}$ are required; these could not be done with the present system).

(3) At a fixed laser-pulse energy, the photoenhanced $\eta / N_{a}$ increases with $N_{a}$ for low $(<5 \mathrm{~Pa})$ partial pressures and, for a fixed $N_{a}, \eta / N_{a}$ increases with increasing $\mathrm{N}_{2}$ pressure in a manner similar to that of a three-body electron attachment process ${ }^{1,7}$ (i.e., the capturing species requires collisional stabilization by $\mathrm{N}_{2}$ ).

(4) In the high- $(E / N)$ region $\left(E / N=3.68 \times 10^{-17} \mathrm{~V}\right.$ $\mathrm{cm}^{2}$ in Fig. 3; see also Fig. 2), where $\eta / N_{a}$ is substantially due to ground-state dissociative attachment, $\eta / N_{a}$ is only slightly dependent on the $\mathrm{N}_{2}$ pressure. In this $E / N$ region, $\eta / N_{a}$ is almost (Fig. 3) independent of the laserpulse energy in the case of single pulse; it varies slowly with the pulse energy in the double-pulse experiments. Conversely, in the low- $(E / N)$ range $(E / N=0.153$ $\times 10^{-17} \mathrm{~V} \mathrm{~cm}{ }^{2}$ in Fig. 3; see also Fig. 2), where $\eta / N_{a}$ is exclusively due to photoenhanced attachment, $\eta / N_{a}$ increases greatly with increasing $\mathrm{N}_{2}$ pressure [see (3) above] and also with the laser-pulse energy for both the single pulse and, especially, the double pulses.

While the $\eta / N_{a}$ data in Figs. 2 and 3 were taken with the 249-nm laser line, other laser wavelengths were also used. Briefly, at the 308-nm laser line whose energy lies at or just below $S_{1}$ there is very little laser absorption and, hence, little photoenhanced attachment up to laserpulse energies of $\sim 1 \mathrm{~mJ}$. For laser light at 222 and 193 $\mathrm{nm}$ for which the molecule can photodissociate, the laser absorption coefficients are large, and the photoenhanced

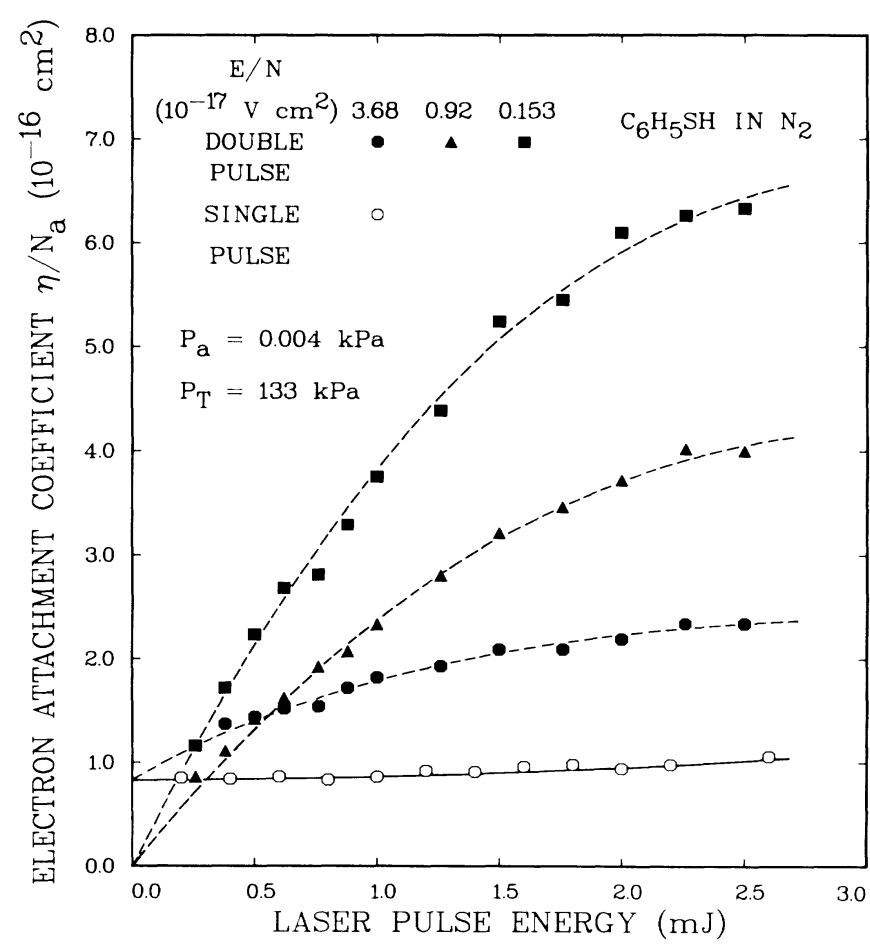

FIG. 3. Electron-attachment coefficient, $\eta / N_{a}$, vs laser-pulse energy at $\lambda=249 \mathrm{~nm}$ for single-pulse and double-pulse $\left(\tau_{d}\right.$ $=12.5 \mathrm{~ms}$ ) irradiation at three $E / N$ values. At $E / N$ $=0.153 \times 10^{-17} \mathrm{~V} \mathrm{~cm}^{2}, \eta / N_{a}$ is predominantly due to photoenhanced attachment, and at $E / N=3.68 \times 10^{-17} \mathrm{~V} \mathrm{~cm}$, $\eta / N_{a}$ is due predominantly to ground-state dissociative attachment (see Fig. 2).

attachment is small (one-fifth to one-half in the laserpulse energy range $0.2-0.6 \mathrm{~mJ}$ ) compared with that due to the 249-nm line under identical conditions, indicating competition between photodissociation and internal conversion to $S_{1}$. This laser-wavelength dependence of the photoenhanced attachment rules out dissociative attachment to vibrationally excited photofragments as the source of the photoenhanced attachment. Laser photoionization is also ruled out as the source of the photoenhanced ion production in view of the observed dependence of the photoenhanced attachment on $E / N$ and the observed greatly enhanced attachment for the second laser pulse. Furthermore, because the experimental arrangement employed for the measurement of $\eta / N_{a}$ cannot distinguish between positive and negative ions (both contribution to the observed signal), we devised a new two-field drift technique with which the positive and negative changes can be separately detected. With this arrangement we confirmed that at the laser-pulse energies employed in this study, the contribution of positive ions (due to laser photoionization) to the signal used to determine $\eta / N_{a}$ is negligibly small except at very low $E / N$ values where it could cause an increase of $\lesssim 10 \%$ in $\eta / N_{a}$. The $\eta / N_{a}$ values in Fig. 2 have been corrected for 
this effect.

It should be noted that the photoenhanced $\eta / N_{a}$ in Figs. 2 and 3 are actually lower limits to their true values since $\eta$ was divided by the number density, $N_{a}$, of the unexcited $\mathrm{C}_{6} \mathrm{H}_{5} \mathrm{SH}$ molecules which is larger than that, $N_{a}^{*}$, of the excited molecules in $T_{1}$. For the lowest laser energy $(0.05 \mathrm{~mJ})$ used for which no saturation effects are expected (Fig. 3), we estimate that $\eta / N_{a}^{*}$ is about 200 times larger than the values of $\eta / N_{a}$ given in Fig. 2 (curve 4).

The present results clearly suggest that laser irradiation can be employed to produce (by strong absorption to an allowed electronic state followed by fast internal conversion to a long-lived state) large numbers of long-lived electronically excited molecules which attach electrons with cross sections $10^{5}-10^{6}$ times larger than the respectively unexcited species.

This research was sponsored in part by the Office of Scientific Research, U. S. Air Force, under interagency agreement DOE No. 40-1523-84 and in part by the Office of Health and Environmental Research, U. S. Department of Energy, under Contract No. DE-AC05840R21400 with Martin Marietta Energy Systems, Inc.

(a) Also at Department of Physics, The University of Tennessee, Knoxville, TN 37996.

(b) Department of Physics, University of Ioannina, Ioannina, Greece.

(c) Also at The National Hellenic Research Foundation, 48 Vassileos Constantinou Avenue, Athens 501/1, Greece.

${ }^{1}$ Electron-Molecule Interactions and Their Applications edited by L. G. Christophorou (Academic, New York, 1984), Vols. 1 and 2.
${ }^{2}$ C. L. Chen and P. J. Chantry, J. Chem. Phys. 71, 3897 (1979).

${ }^{3}$ I. M. Beterov and N. V. Fateyev, J. Phys. (Paris), Colloq. C7-447 (1983)

${ }^{4}$ M. J. Rossi, H. Helm, and D. C. Lorents, Appl. Phys. Lett. 47, 576 (1985)

${ }^{5}$ P. D. Burrow, J. Chem. Phys. 59, 4922 (1973); D. S. Belic and R. I. Hall, J. Phys. B 14, 365 (1981).

${ }^{6}$ C. Bottcher and B. D. Buckley, J. Phys. B 12, L497 (1979).

${ }^{7}$ L. G. Christophorou, Atomic and Molecular Radiation Physics (Wiley-Interscience, New York, 1971), p. 441; S. R. Hunter and L. G. Christophorou, J. Chem. Phys. 80, 6150 (1984).

${ }^{8}$ S. R. Hunter, J. G. Carter, and L. G. Christophorou, J. Appl. Phys. 60, 24 (1986).

${ }^{9}$ Organic Molecular Photophysics, edited by J. B. Birks (Wiley-Interscience, New York, 1975), Vol. 2.

${ }^{10}$ S. P. McGlynn, T. Azumi, and M. Kinoshita, Molecular Spectroscopy of the Triplet State (Prentice-Hall, New Jersey, 1969), p. 40.

${ }^{11}$ P. M. Rentzepis, Science 129, 239 (1970).

${ }^{12}$ In these studies care must be taken to ensure that photodissociation-a laser-wavelength- and laser-pulse-energy-dependent process-does not occur, or if it does occur, that it is properly studied and considered.

${ }^{13}$ H. H. Jaffé and M. Orchin, Theory and Applications of Ultraviolet Spectroscopy (Wiley, New York, 1962), p. 477.

${ }^{14}$ P. G. Russell, J. Phys. Chem. 79, 1347 (1975).

${ }^{15}$ L. G. Christophorou, D. L. McCorkle, and J. G. Carter, J. Chem. Phys. 60, 3779 (1974); A. Modelli, D. Jones, F. P. Colonna, and G. Distefano, Chem. Phys. 77, 153 (1983).

${ }^{16}$ Such triplet excimers have been proposed [e.g., see Photochemistry, edited by D. Bryce-Smith (The Chemical Society, London, 1975), Vol. 6, pp. 198,199; T. F. Hunter and M. G. Stock, Chem. Phys. Lett. 22, 368 (1973), and J. Chem. Soc. Faraday Trans. 2 70, 1028 (1974)] to interpret a number of photophysical processes in organic (e.g., benzene) vapors. 